\title{
Three Kinds of Optical Flow Technique: LCT, DAVE and DAVE4VM
}

\author{
Jihong Liu ${ }^{1,}$,, Yanhui Dong ${ }^{1}$ and Yuhong Zheng ${ }^{2}$ \\ ${ }^{1}$ Shijiazhuang University, Shijiazhuang, 050035, China \\ ${ }^{2}$ Shijiazhuang Post and Telecommunications Technical College, Shijiazhuang, \\ 050031, China \\ apljh@163.com
}

Keywords: velocity field, active region, optical flow techniques, helicity

Abstract. Photospheric velocity field is an important parameter in studying solar eruptions. This parameter can be deduced by three kinds of optical flow techniques, i.e., LCT ${ }^{[1]}$, DAVE and DAVE4VM $^{[2]}$. By using an example of active region (AR) NOAA 12017, we have compared the distribution of the velocity field and magnetic helicity transport rate which calculated from the velocity field by these three methods. It is found that, the velocity fields are very similar for the results from LCT and DAVE method, while much different for those from DAVE4VM. However, the helicity transport rates are in similar trend for the results from all methods.

\section{Introduction}

The optical flow techniques refer to a series of new image analyzing techniques arisen recently on the researches of solar magnetic non-potentiality. The most popular ones are LCT (local correlation tracking), DAVE (differential affine velocity estimator) and DAVE4VM (differential affine velocity estimator for vector magnetograms). Benefit from the optical flow techniques, the transverse velocity field of the magnetic features on the solar surface can be determined by a time sequence of high-quality images currently produced by high-resolution observations either from the ground or in space. Consequently, the magnetic helicity flux which represents the non-potentiality of the AR, could be acquired. Former works have shown that they are probably related closely to the solar eruptionss, such as flares, filament eruptions and coronal mass ejections ${ }^{[3]}$. Using the line-of-sight magnetic fields and the vector magnetic fields based on a vector magnetogram series from the Helioseismic and Magnetic Imager (HMI) on board the Solar Dynamic Observatory (SDO) ${ }^{[4]}$, the velocity filed of NOAA active region 12017 has been calculated by these methods. By comparing the velocity field by different methods, the merits and disadvantages of these methods are clearly shown.

The work is arranged as follows: (1) The velocity fields by different optical flow techniques. (2) The helicity flux rate by different optical flow techniques. (3) Conclusions and discussions.

\section{Velocity Fields by Different Optical Flow Techniques.}

We use line of sight and vector magnetic field data taken by HMI ${ }^{[3,5]}$ to derive the photospheric flow field. The HMI instrument is a filtergraph with full-disk coverage of $4096 \times 4096$ pixels. The spatial resolution is about 1 " with a 0.5 "pixel size. The spectral line is the Fe I $6173 \AA^{[4,6]}$.

Fig 1. Shows the distribution of the velocity field deduced by LCT (left) and DAVE technique (right). Both methods use the line of sight magnetic field. It is found that, the distribution of the velocity field is very similar for the results from LCT and DAVE method.

Fig 2. Shows the distribution of the velocity field deduced by the DAVE4VM technique. It is much different from those by LCT and DAVE method. The DAVE4VM method estimates a field aligned plasma velocity from only magnetic field observations, and the DAVE produces a biased estimation of the total horizontal plasma velocity ${ }^{[2]}$. So we need to analyze the difference between the magnetic helicity derived from different velocity field. While magnetic helicity transport rate is a very import non-potentiality parameter and relate closely with solar eruptions. 

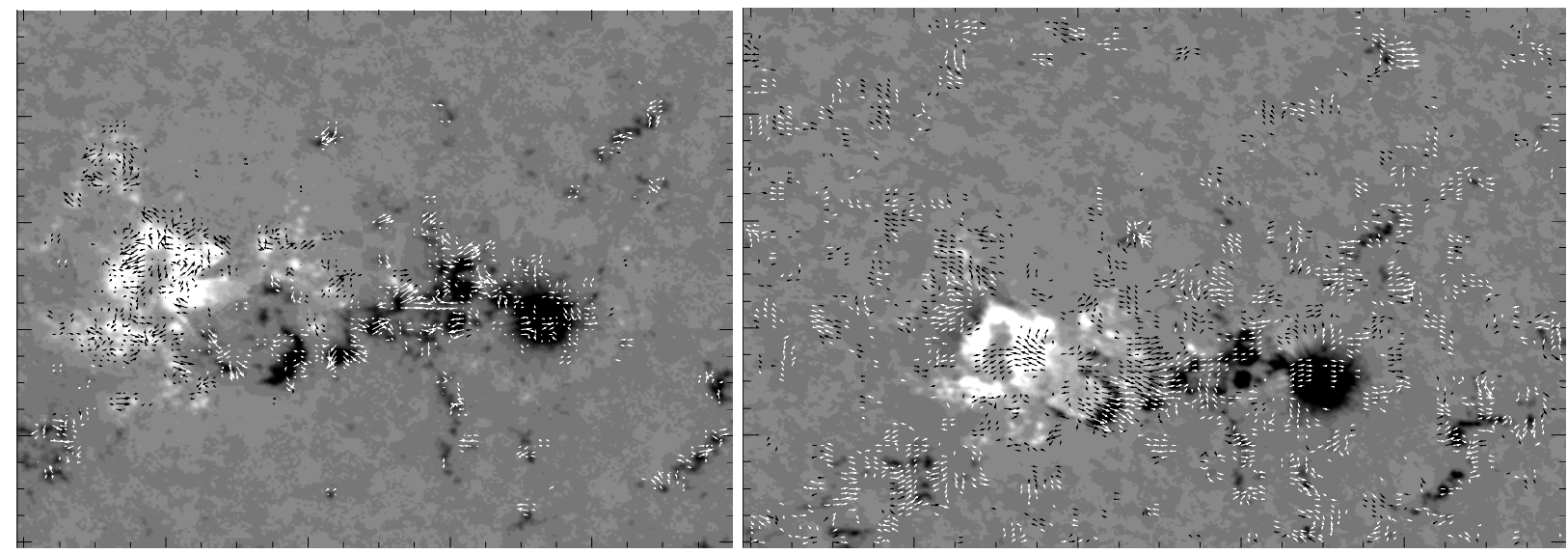

Fig. 1 Velocity field by LCT technique (left) and DAVE technique (right), the grey-scale map are the vertical magnetic field in active region AR 12017 at 18:00 UT, of 24 March 2014. Black and white refer to negative and positive fields, respectively. The arrows mark the horizontal velocity fields.

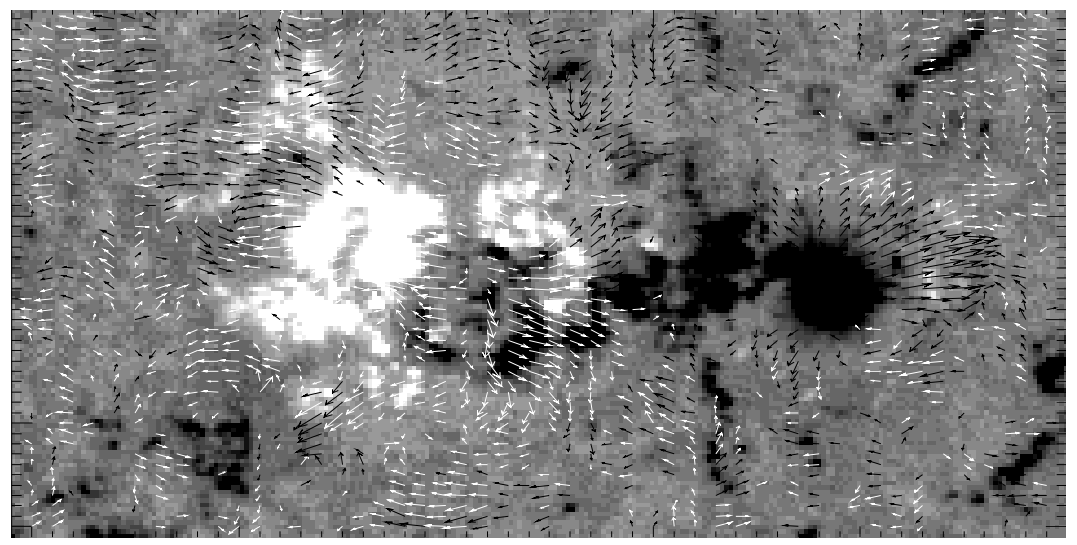

Fig. 2 Similar to Fig. 1, but for velocity field by DAVE4VM technique.

\section{Helicity Flux Rate by Different Optical Flow Techniques.}

The transport rate of magnetic helicity from the sub-photosphere to corona by the photospheric horizontal motions is described by the equation

$$
\frac{d H}{d t}=2 \rrbracket\left(\vec{A}_{p} \cdot \vec{B}_{t}\right) V_{\perp n} d S-2 \rrbracket\left(\vec{A}_{p} \cdot V_{\perp n}\right) B_{n} d S
$$

where $A_{p}$ is the vector potential of the potential field $B_{p}, B_{t}$ and $B_{n}$ denote the tangential and normal magnetic fields, and $\mathrm{V}_{\perp} \mathrm{t}$ and $\mathrm{V}_{\perp_{\mathrm{n}}}$ are the tangential and normal components of velocity $\mathrm{V}_{\perp}$, the velocity perpendicular to the magnetic field lines.

By introducing the flux transport velocity

$$
\vec{u}=v_{\perp t}-\left(v_{\perp n} / B_{n}\right) B_{t}
$$

$\mathrm{D}^{\prime}$ emoulin \&Berger ${ }^{[7]}$ simplified Equations (1):

$$
\frac{d H}{d t}=-2 \int\left(\vec{A}_{p} \cdot \vec{u}\right) B_{n} d S
$$

So the helicity flux can be computed from time series of line-of-sight magnetograms.

When using LCT method, we follow Chae et al. (2001) to choose the FWHM of the adopting function as 8 "and the time interval as 1 hour, and to set the horizontal velocity in regions with low flux density $(<10 \mathrm{G})$ or low cross-correlation value $(<0.9)$ to zero to reduce the noise effects ${ }^{[1,8]}$. When 
using the DAVE and DAVE4VM, we follow Liu et al. (2013) to choose the FWHM of the adopting function as 19"and the time interval as 720 second (12 minutes) ${ }^{[6]}$, and to set the horizontal velocity in regions with low flux density (line of sight magnetic field $<10 \mathrm{G}$ and horizontal magnetic field $<50 \mathrm{G}$ ) to zero to reduce the noise effects.

Fig. 3 shows the time evolution of helicity flux. The left-up panel shows the result deduced by LCT method, and the left-down panel shows the result deduced by DAVE method. Both methods use the line of sight magnetic field with time resolution of one hours of HMI/SDO. It can be seen obviously that the evolution trend is similar for them. The right-up and the right-down panel is the result deduced by the DAVE method and DAVE4VM technique with the vector magnetic field of HMI/SDO of the time resolution of 12 minutes, respectively. It can be seen that the time evolution trend are much similar for them, also. The different value of helicity may mainly due to the different field-of-view selected for different method when using the magnetic field data. And it can be seen also that the time resolution is not critical for the helicity evolution trend.
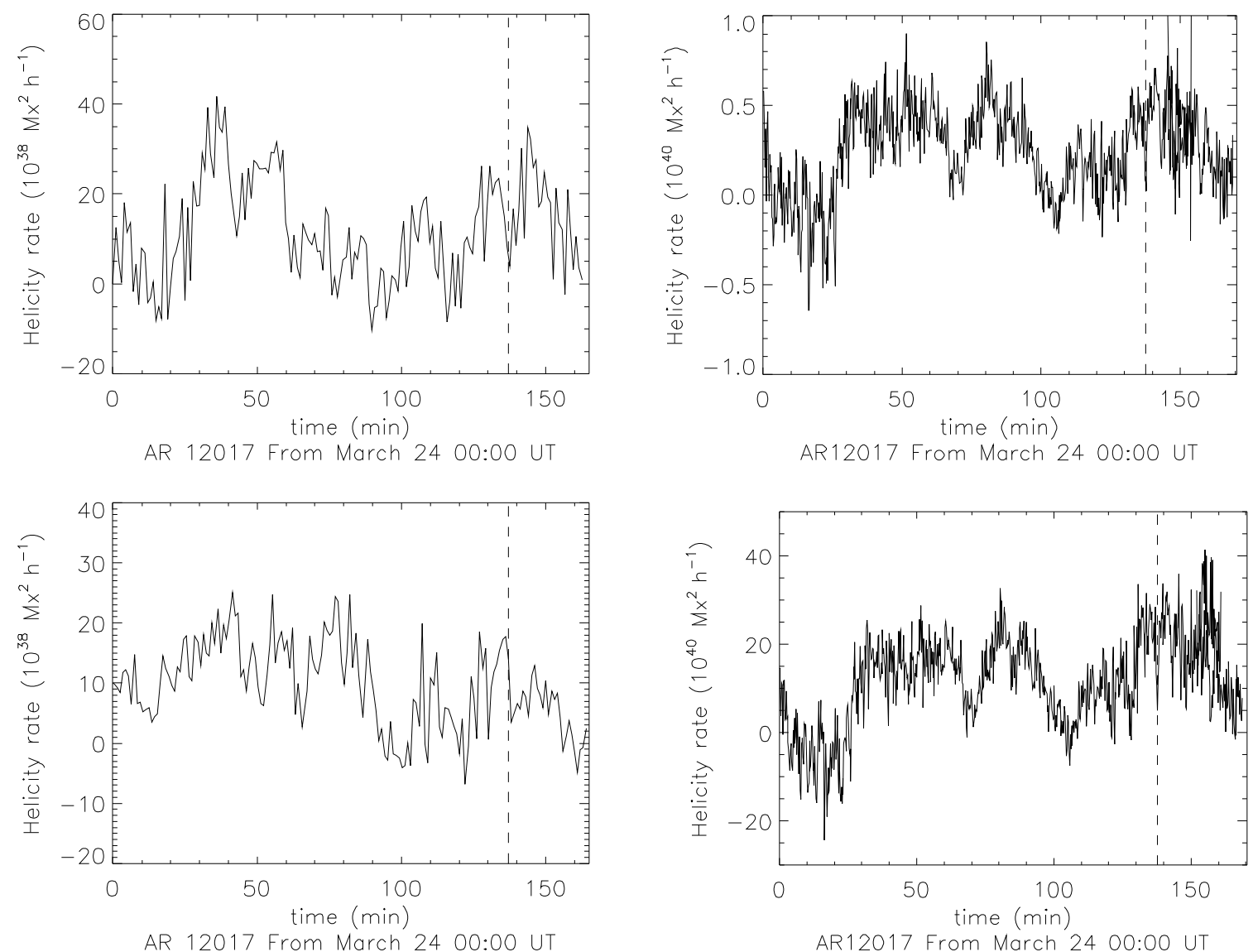

Fig. 3 Time evolution of helicity flux by LCT (left-up panel), DAVE(right-up panel),DAVE for 1 hour time resolution data (left-down panel) and DAVE4VM technique (right-down panel). The dashed vertical line mark the $\mathrm{X}$-class flare time.

technique which both using the vector magnetic field of HMI/SDO with the time resolution of 12 minutes. It can be seen that the time evolution trend are much similar for them, also. The different value of helicity may mainly due to the different field-of-view selected for different method when using the magnetic field data. And it can be seen also that the time resolution is not critical for the helicity evolution trend. 


\section{Conclusions and discussions}

The velocity filds derived from time series of magnetic field observed by HMI by three optical flow techniques are compared by using an example of AR NOAA 12017. It is found that the velocity fields are very similar for results deduced from LCT and DAVE method, while much different for those deduced from DAVE4VM. Further, the helicity flux rate deduced by three different optical flow methods were compared and analyzed, too. It is found that the helicity transport rates are in similar trend for the results deduced from all methods.

Liu et al. $2013^{[4]}$ mentioned that although the time revolution trend is very similar for DAVE/LCT and DAVE4VM as a whole, the former two underestimated the emerging component of $\mathrm{H}$ about $20 \sim 30 \%$ than that of the latter. For AR NOAA 12017, it is about 10\%. Some previous work represented that after the flare, the increasing rate of the helicity flux decreased, which may imply the energy and complexity transportation slowed down after the flare ${ }^{[9,10]}$. Our results for AR NOAA 12017 confirm the former argumentation.

\section{Acknowledgements}

This work is supported by grants 11178012,11078016 of National Natural Sci-ence Foun-dation of China, A2010001942, A2011210017, A2014110008 of HeBei Natural Sci-ence Foundation of China, and XJPT002 of Shijiazhuang University, 13040449 of the Twelfth Five-Year Plan of Hebei Province. Thanks the refree for her useful advise.

\section{References}

[1] J.,Chae, ApJ, 560: L95.(2001)

[2] P.W.,Schuck, ApJ,683:1134. ( 2008).

[3] P.H., Scherrer, J. Schou, R.I. Bush, et al., SoPh., 275, 207S. (2012)

[4] Y. Liu, J. Zhao, P.W. Schuck, Solar Phys. 287, 279.(2013)

[5] J. Schou, P.H. Scherrer, R.I. Bush, et al. ,Solar Phys, 275,229S. (2012)

[6] Y. Liu, P.W. Schuck, ApJ. 761, 105.(2012)

[7] P. D' emoulin, and M.A. Berger, Solar Phys. 215, 203 (2003).

[8] J. Chae, Y.J. Moon, Y.D. Park, Solar Phys. 223, 39.(2004)

[9] J.H. Liu, et al. 2013 International Conference on Frontiers of Energy, Environmental Materials and Civil Engineering , 455, (2013)

[10] Y. Zhang et al. ApJ, 704, 1622 (2009). 